PostGRAD. MED. J. (1964), 40, 692

\title{
OPTIC ATROPHY
}

F. Clifford Rose, M.B., M.R.C.P.

Consultant Neurologist, Royal Eye Medical Ophthalmology Unit, London, S.E.1.

\section{BASIC CONSIDERATIONS}

Optic atrophy is a misleading term which, embedded by tradition and usage, resists precise definition. It is used when there is excessive pallor of the optic disc and is thus a physical sign and not a diagnosis. Since there are many causes of optic pallor, the term is usually reserved for those cases in which there is associated visual defect, either of fields or acuity, indicating a degeneration of the fibres of the optic nerve. This definition is not satisfactory since not only may visual acuity be normal in spite of optic pallor, but degeneration of the optic nerve can be sufficient to cause diminished visual function but without optic pallor, e.g., in traumatic lesions of the nerve, blindness being immediate but pallor not developing for perhaps three weeks. Pallor is thus not the only criterion of optic atrophy and to be certain of degeneration of the optic nerve the following are also necessary:-

1. Diminished visual acuity.

2. Visual field changes.

3. Recession of the disc.

When there is doubt as to whether a disc is pathologically pale the knowledge of diminished visual function is often used to decide on the presence of optic atrophy.

\section{The Cause of Pallor}

There are three possible causes for optic pallor, viz: -

1. Overgrowth of glial tissue.

2. Degeneration of nerve fibres.

3. Diminished amount of blood at the disc.

1. Although there are glial cells in the optic nerve, gliosis would not be visible through the lamina cribrosa. There are also supporting cells in the retina, and it is here that gliosis may be seen, particularly following papilloedema where there is filling in of the physiological cup with exudate in addition to an increase in glial tissue.

2. The optic disc is whiter than the surrounding fundus because there is neither a highly vascular choroidal coat nor pigment epithelium in this area. Loss of medullary sheaths cannot be a cause of optic pallor since the medullary sheaths normally end as the fibres pierce the lamina cribrosa which, since it is opaq家e, prevents the medullary sheaths from being visible. Furthermore, when medullated fibres seen funduscopically as a congenital anomaly are demyelinated, they become not whiter byt transparent. At the disc the only retinal lager present is the nerve fibre layer and it is \&egeneration of this layer that probably produees the lack-lustre appearance of the atrophic disc.

3. That diminished vascularity can cause optic pallor may be confirmed by compressing the eye: at the time that pulsation of the retidal artery ceases the disc becomes pale. Both the disc and retina may be paler than normal because of a generalised anæmia. When any tissue of the body degenerates there is a loss of blood supply to it; this is true of the ogatic nerve head, and there is little doubt that most satisfactory explanation of pallor in sptic atrophy is diminution of the number of laries to the disc.

\section{Anatomy of the Optic Nerve Head} 항

The optic nerve head, named "papilla" \$by Briggs in 1688 (mistakenly, since it does project forwards), is slightly oval, approximatioly $1.5 \mathrm{~mm}$. in diameter with the longer a is vertical. The blood supply of the optic negue and nerve head is extremely complex. The peripheral fibres in the optic nerve are supplied from vascular plexi of the surrounding meninges, viz., perforating vessels from the dura mater which in their turn arise from branches of the anterior cerebral artery and ophthalmic artexy, vessels from the pia mater arising from the external carotid artery. The central fibres arre supplied by the anterior and posterior recurrent arteries from the ophthalmic artery, which gives no perforating branches. Anterior ramifieations of these anastomose with the Zinn-Hafer circle. The central retinal artery supplies retina but not the optic nerve. With a central retinal artery occlusion, the optic nerve sifll has its blood supply, and so the disc is gुot immediately white.

The number of vessels seen crossing the disc margin has been used as a test of atrop By. Larger vessels which are obvious arteries or 
veins (adding up to about nine, normally) are excluded and only those smaller vessels which are not obviously arterial or venous counted; these amount to about ten with little variation from observer error. The capillary count correlates with both visual acuity and the visual field.

\section{Physiology}

The optic nerve is not a peripheral nerve but a fibre tract comparable to the white matter of the brain from which it is embryologically derived. Its constituent nerve fibres arise in the ganglion cell layer of the retina and end in the lateral geniculate body. The visual cell can be equated with the sensory organ, the nucleus of the bipolar cell with the posterior root ganglion and the ganglion cells with the cuneate and gracile nuclei. Alternatively, the retina can be regarded as modified cortex, since they are both derived from the same embryonic tissue and they may both undergo the same pathological change, e.g. Tay-Sachs' disease. The optic nerve, like the fibre tracts of the central nervous system (CNS), cannot regenerate as it has no neurolemmal sheath containing Schwann cells, and although there is an attempt at regeneration, this always fails. Experimental section of the optic nerve produces pallor usually within fourteen days and certainly within three months, and the pallor will be more marked the nearer the section is to the bulb. Attempts at regeneration occur from both ends of the cut nerve indicating that there are centrifugal fibres from the brain. There is no doubt that these exist in animals, e.g., the cat, where ciliary function is controlled by centrifugal fibres in the optic nerve. In more primitive animals, e.g., the newt, regeneration of the cut optic nerve is successful.

The optic nerve contains about one million fibres of varied thickness; the histogram of fibre size corresponds to that of the brain and spinal cord, viz., from $0.7-8.6 \mu$ but most are under $1 \mu$. The larger ganglion cells are at the periphery of the retina and these give rise to thicker axons; the larger number of small fibres at the macula is related to differentiation of central vision.

\section{Clinical Considerations}

\section{Incidence of Optic Atrophy}

Between ten and twelve thousand new blind certificates are issued each year. The majority of these-approximately $70 \%$ - are for patients over the age of 70 years. Because the blind registered have a much higher mortality than the average population (twenty times as much) they average about 100,000 in any one year. Although only $4.5 \%$ of these have optic atrophy, in the younger age groups between the ages of $15-41$, optic atrophy is the largest single cause of blindness ( $18.5 \%$ of certificates).

\section{Classification}

No single satisfactory classification has been devised, but each of the following may prove useful.

\section{Ophthalmoscopic}

A. If the disc margin is clearly delineated and the disc dead white, the optic atrophy is called "primary"; if the margin is blurred and the disc a greyish colour, "secondary". Although this distinction is valuable the nomenclature is confusing because of the varying usage, some assuming "secondary" to mean consecutive to papilloedema and others applying the term to optic atrophy that is not due to a lesion of the nerve itself. Provided this classification is used for descriptive and not ætiological purposes, it can be helpful.

B. The pallor may extend over the whole disc - total optic atrophy-or may be marked over a segmental area, e.g., in trauma or in retrobulbar neuritis where there is temporal pallor because the macular fibres are selectively damaged. The disc itself may show greater than normal cupping which, if extreme as in glaucoma, is sometimes called "cavernous".

\section{Physiological}

If the primary lesion is in the ganglion cells of the retina, then the optic atrophy is called ascending, and if in the optic nerve fibres, descending. Descending degeneration is slower than ascending degeneration. Although elsewhere in the CNS a degenerating cell does not affect the cell with which it synapses, transsynaptic degeneration does occur in the visual pathway, e.g. a lesion of the neuroepithelial layer of the retina will be followed by optic atrophy and atrophy of the lateral geniculate body.

\section{Anatomical}

Optic atrophy often follows optic neuritis, which can be divided depending on the part of the nerve affected, either in a longitudinal or transverse plane.

\section{A. Longitudinal}

Optic neuritis is divided into retrobulbar neuritis if the optic nerve head is not involved, papillitis if it is, and neuro-retinitis if there is spread to the retina. With a neuro-retinal lesion, the scotoma is often to colours, especially blue, indicating a less severe loss (since colour provides a less intense stimulus). The scotoma is 
either central or centro-cæcal and complete blindness (i.e., failure to perceive light) is uncommon.

B. Transverse

1. Perineuritis.

2. Axial retrobulbar neuritis, which means that the central macular fibres are involved producing a central scotoma.

3. Total transverse neuritis if there is complete blindness.

("Chronic retrobulbar neuritis" is the older term for toxic amblyopia and should be discarded). 4. Aetiological

A. Inflammatory, e.g., retrobulbar neuritis

B. Compressive, i. by tumour

ii. by bone, e.g., cranio-

iii. by blood vessel stenosis

- internal carotid artery

- ophthalmic artery

- aneurysm

iv. in glaucoma

v. after papillodema

C. Congenital and genetic, e.g., Leber's.

D. Vascular, a. central retinal artery occlusion;

b. arteriosclerotic.

E. Deficiency, e.g., $\mathbf{B}_{12}$ in subacute combined degeneration of spinal cord, nutritional.

F. Toxic.

G. Traumatic.

H. Metabolic, e.g., Tay-Sachs' disease.

I. Associated with retinal lesions, e.g., retinitis pigmentosa.

\section{J. Others.}

Since a consideration of all these is outside the scope of the present paper, only some more common examples of the first three will be discussed.

\section{Retrobulbar Neuritis}

This presents in either sex between the ages of 15-45 years with blurred vision in one eye; about $10 \%$ of cases are bilateral. The field defect is usually a central scotoma which is described by the patients as not seeing directly in front, but only around an object. Tenderness or pain in the globe, especially on movement, is common. The visual acuity deteriorates for a few hours or days and then improves, so that the majority recover $6 / 6$ vision within twelve weeks, at which time temporal pallor may be seen. Two other signs often found are ill-sustained pupillary contraction to light, and lowering of dark adaptation; the former may occur in normal subjects but can be a useful additional sign. Loss of macular reflex is not demonstrable in retrobulbar neuritis, and is more often seen in ascending atrophy främ a retinal lesion. There is a great variability both as to extent of visual loss, degree 8 of recovery, and fundus change, but the clinieal pattern is usually easily recognised. Occasionally, there is a history of transient visural deterioration following food, exercise or a tot bath.

This pattern of symptoms and signs is dipe to a plaque of demyelination in the optic nefre with surrounding odema. This compresses the central and thinner macular fibres, interferitg with nervous conduction to produce a central scotoma. The odema resolves allowing a gơod deal of recovery but leaving a small glietic plaque with sufficient degeneration of macufar fibres to produce temporal pallor. The $p$ is due to the stretching of the meningeal vessels over the œedematous optic nerve and aggravatiân by eye movement is due to pulling of the external ocular muscles on the fibrous zonfe of Zinn which is contiguous with the meninges overlying the optic nerve.

If toxic and deficiency causes are excluded, then optic neuritis is most often due to dempelinating disease. This does not mean that every case will be followed invariably by disseming CNS lesions, but the process in the optic berye is the same as that which occurs in the certegal nervous system in disseminated sclerosis. ज्The percentage of cases of retrobulbar neuritis that later develops disseminated lesions depends on the length of follow-up and varies from 34 $66 \%$. Not all cases of retrobulbar neuritis followed by temporal pallor, but the incidenge of temporal pallor in cases of D.S. increases from $37 \%$ in the first year to over $50 \%$ in Pe $^{2}$ fifth year. Autopsies on cases of D.S. will show involvement of the optic nerve even though ocular symptoms have not been magifest during life.

In children there is a greater frequency iff papillitis which has a worse prognosis but bilateral cases are commoner in this age groop and these have a more favourable prognosis. Neither the rapidity of onset nor the occurrenge of pain has any bearing on prognosis.

\section{Compressive}

Since this group includes cases which $\stackrel{\circ}{\mathrm{a}} \mathrm{be}$ remediable, care must always be taken to \&8:clude a tumour. A central scotoma "breakinig through" to the periphery is highly suggestive of optic nerve compression. Other types \&f field defects are also characteristic but $\mathrm{nB} \mathrm{t}$ pathognomonic, e.g., in chiasmal compression hemianopia is characteristic but a central scof- 
oma may also occur. Plain X-ray of the skull and optic foramina are not sufficient and contrast radiography and other special techniques may be indicated. Even when these are negative, exploratory craniotomy may reveal a benign tumour, e.g., meningioma, and its removal may be followed by a recovery of vision.

\section{Congenital and Genetic}

This group accounts for most cases of optic atrophy in children in this country as in other parts of the world, and it may be classified as follows: -

1. Congenital-One of the diagnostic difficulties in children is that the disc is normally paler than in the adult, and the differential diagnosis will include hypoplasia which is usually easy to differentiate as the discs are smaller.

2. Associated with hydrocephalus-Between the ages of six months and six years about $50 \%$ are in this group.

3. Hereditary optic atrophy is commonly dominant, occasionally recessive, and only rarely fits in with the group described by Leber.

4. Leber's disease-Although von Graefe had earlier described the syndrome, Leber delineated the entity in 1871 by describing 55 cases in 16 families. He found $90 \%$ of those affected were male, and that it occurred between the ages of 18 and 23. Little had been added to Leber's original description, although in Japan the sex incidence is nearly equal-3 males: 2 females. The onset is rapid and asymmetrical, involving both eyes within a few days to six months. In the early stages there is a fleeting papillitis giving a central scotoma, but peripheral constriction alone also occurs. The maximum disability is within two months and it rarely progresses further after six months. Complete blindness is exceptional and slight improvement may occur, especially in the young.
It is a relatively rare hereditary disease; the mode of inheritance is unknown, but is not strictly Mendelian. Transmission is through the unaffected female, and a history of consanguinity is uncommon. If it were transmitted as a true sex-linked recessive, as in colour blindness or haemophilia, the incidence would be 200 males: 1 female.

Retinal lesions occur, colour blindness is frequently present and nystagmus and vertigo have been reported.

Although there are over 1,000 cases in the literature, there have been only three patholological reports and in one of these the CNS was not examined. All cases revealed loss of medullary sheaths in the papillo-macular bundle with glial overgrowth. The ganglion cells and nerve fibre layer were atrophied and there was a moderate degree of atrophy of the inner nuclear layer.

5. Associated with CNS disease-In Marie's cerebellar ataxia optic atrophy is common, shows itself early and affects chiefly the male. Where optic atrophy is transmitted by affected males it is associated with retinal lesions. In this condition, as with Charcot-Marie-Tooth disease (peroneal muscular atrophy), the inheritance is dominant. In Friedreich's ataxia, in which optic atrophy is uncommon, and hypertrophic polyneuritis, the mode of inheritance is recessive and in Pelizeus-Merzbacher syndrome it is sex-linked. Familial spastic paraplegia and areflexia are other conditions in which optic atrophy occurs.

\section{Conclusion}

The gravamen of this paper is that optic atrophy is a physical sign and not a diagnosis. Patients showing this sign must have a complete genetic history, a thorough examination and full neurological assessment. 